Gunther 0. Hofmann

BG-Unfallklinik Murnau

\title{
Therapeutische Optionen bei persistierendem Kniegelenkinfekt
}

$F$

\section{Zusammenfassung}

Die erfolgreiche Behandlung eines Kniegelenkinfekts ist eine Funktion des Faktors Zeit. Beim Vorliegen eines Frühinfekts $(<6$ Wochen) kann die Behandlung zunächst durch arthroskopische Lavagen in kurzen 2- bis 3-tägigen Intervallen erfolgen. Nur bei Infektpersistenz ist das offene Vorgehen indiziert. Beim Spätinfekt ( $>6$ Wochen) sollte sofort offen vorgegangen werden. Auch hier empfiehlt sich ein standardisiertes Revisionsprotokoll mit kurzen Intervalleingriffen. Eine testgerechte, systemische Antibiose ist begleitend zu empfehlen. Bei frühzeitigem Behandlungsbeginn lassen sich Gelenkinfektionen nahezu immer beherrschen, wobei dennoch häufig ein deutliches Bewegungsdefizit zurückbleibt. Spätinfekte führen fast immer zum ausgedehnten Gelenkbinnenschaden oder sogar zum Gelenkverlust. Als therapeutische Optionen für den infektbedingten Gelenkverlust kommen in abnehmender Priorität in Frage: Endoprothetik, Arthrodese und Resektionszustand. Eine Altersgrenze für die Endoprothetik nach infektbedingtem Gelenkverlust existiert u. E. nicht, weder nach oben noch nach unten. Voraussetzung ist allerdings, dass die Infektion mit ausreichender Wahrscheinlichkeit beherrscht ist.

\section{Schlüsselwörter}

Gelenkinfektion · Kniegelenk · Akut und chronisch · Arthroskopie · Endoprothetik ür die großen Gelenke des menschlichen Körpers werden akute und chronische Infektionen in unterschiedlicher Häufigkeit beschrieben. Dabei steht das Kniegelenk mit fast 3/4 aller Infektionsfälle ganz klar im Vordergrund (Tabelle 1). Ursache für diese hohe Prävalenz an Gelenkinfektionen dürfte nicht so sehr die Tatsache sein, dass das Kniegelenk das mechanisch am stärksten beanspruchte Gelenk des menschlichen Körpers ist, sondern dass diagnostische und therapeutische Maßnahmen (Punktionen, intraartikuläre Injektionen, $\mathrm{Ar}$ throskopien) an diesem Gelenk weitaus am häufigsten durchgeführt werden.

\section{Klassifikation}

Die Stadien einer Gelenkinfektion können

1. nach dem morphologischen Korrelat, also nach den bereits vollzogenen anatomisch-histologischen Veränderungen,

\begin{tabular}{|c|c|}
\hline Lokalisation & Häufigkeit [\%] \\
\hline Knie & 73 \\
\hline Ellenbogen & 14 \\
\hline Schulter & 9 \\
\hline Hüfte & 5 \\
\hline
\end{tabular}

2. nach dem zeitlichen Verlauf zwischen Kontamination des Gelenkbinnenraums, Infektions- und Therapiebeginn eingeteilt werden.

\section{Klassifikation nach morphologischem Korrelat}

Diese umfasst die folgenden Stadien:

- Stadium I: Synovitis purulenta

- Stadium II: Gelenkempyem

- Stadium III: Infektpanarthritis

- Stadium IV: Chronische Infektarthrose

\section{Klassifikation nach zeitlichem Verlauf}

Bezüglich des zeitlichen Verlaufs wird dann von einem Frühinfekt gesprochen, wenn zwischen Kontamination bzw. Infektion und Behandlungsbeginn weniger als 6 Wochen vergangen sind, von einem Spätinfekt, wenn zwischen Infektion und Beginn der Therapie mehr als 6 Wochen liegen.

\footnotetext{
๑) Springer-Verlag 2003

Prof. Dr. Dr. Gunther 0. Hofmann Abteilung für Septische und Wiederherstellungschirurgie, BG-Unfallklinik Murnau,

Prof.-Küntscher-Straße 8,82418 Murnau, E-Mail: gunther.hofmann@bgu-murnau.de, Tel.: 08841-482451 oder 08841-482454, Fax: 08841-482117
} 


\section{G.O. Hofmann}

Treatment options

in the presence of persistent knee joint infection

\section{Abstract}

The time factor is of paramount importance in the treatment of an infected knee joint. Repeated arthroscopic revision with irrigation of the infected joint at 2- to 3-day intervals is the best initial procedure for early infections ( $<6$ weeks). Only for a late joint infection ( $>6$ weeks) and in the case of persisting early infection following arthroscopic revisions is an open procedure recommended. A standardized revision protocol with surgery every 2-3 days is combined with a systemic antibiotic therapy following determination of the pathogen(s) concerned and any resistance characteristics. Early knee joint infection can be treated successfully in nearly all cases if treatment is started immediately, though mobility may remain noticeably restricted. Late infections almost always lead to severe destruction of the joint. For treatment following the total loss of a knee joint as a result of infection three different procedures are available: total knee arthroplasty, arthrodesis, and resection, in declining order of priority. We do not recognize any age-dependent threshold for total knee replacement. The only condition is that the infection has been reliably eradicated.

\section{Keywords}

Joint infection - Knee joint · Acute and chronic · Arthroscopy · Total knee replacement

\section{Heilungschancen der verschiedenen Infektstadien}

Eine Synovitis purulenta erfüllt noch die Kriterien der Reversibilität, was bei einem Gelenkempyem meist schon nicht mehr gegeben ist. Während beim Frühinfekt noch eine Chance auf Sanierung und Restitutio ad integrum besteht, gelingt spätestens nach Ablauf des Stadiums II keine Ausheilung mehr. Der Infekt wird chronisch und persistiert, irreversible histopathomorphologisch fassbare Veränderungen schreiten voran. Der Knorpel im Gelenk geht unter, intraartikuläre Bandstrukturen werden zerstört, der subchondrale Knorpel unterzieht sich ebenfalls Veränderungen, er kann sowohl sklerosieren als auch demineralisieren.

Schließlich mündet das Infektgeschehen unweigerlich in den völligen Gelenkverlust, in die chronische Infektarthrose, die dann unbehandelt im für den Patienten günstigsten Fall ankylosiert und somit wenigstens spontan zu einer Schmerzlinderung beitragen kann.

\section{Diagnostik des Gelenkinfekts}

Während die Diagnostik eines akuten frühen Gelenkinfekts noch relativ einfach ist, weil die klinischen Entzündungszeichen im Vordergrund stehen, kann die Diagnosestellung eines chronisch persistierenden, schon lange Zeit larvierend bestehenden Infekts sehr viel schwieriger sein, insbesondere dann, wenn die Infektion durch einen subklinischen Verlauf unter Antibiose u. U. seit Wochen und Monaten besteht.

\section{Anamnese}

Aus der Anamnese lassen sich Schlüsse ziehen über

- eine vorhandene Abwehrschwäche des Patienten (Kortisontherapie, Diabetes, Rheuma, starker Raucher),

- vorangegangene Punktionen des Gelenks und

- intraartikuläre Infektionen sowie

- vorangegangene diagnostische und therapeutische Arthroskopien.

\section{Klinische Diagnostik, Labor und bildgebende Verfahren}

Während dem klinischen Befund mit den klassischen Entzündungszeichen beim Frühinfekt eine Schlüsselrolle zukommt, erweisen sich die klinische Befunderhebung und Untersuchung im Fall der chronischen Infektion oftmals als sehr schwierig.

Blutbild und Serumchemie haben wichtigen Hinweischarakter, jedoch keine beweisende Bedeutung. Sowohl für das C-reaktive Protein als auch für die Blutsenkungsgeschwindigkeit ist die Quote falsch-negativer Laborresultate sehr hoch (17\% bzw. 22\%).

Die konventionelle Röntgendiagnostik kann bei der Aufdeckung des

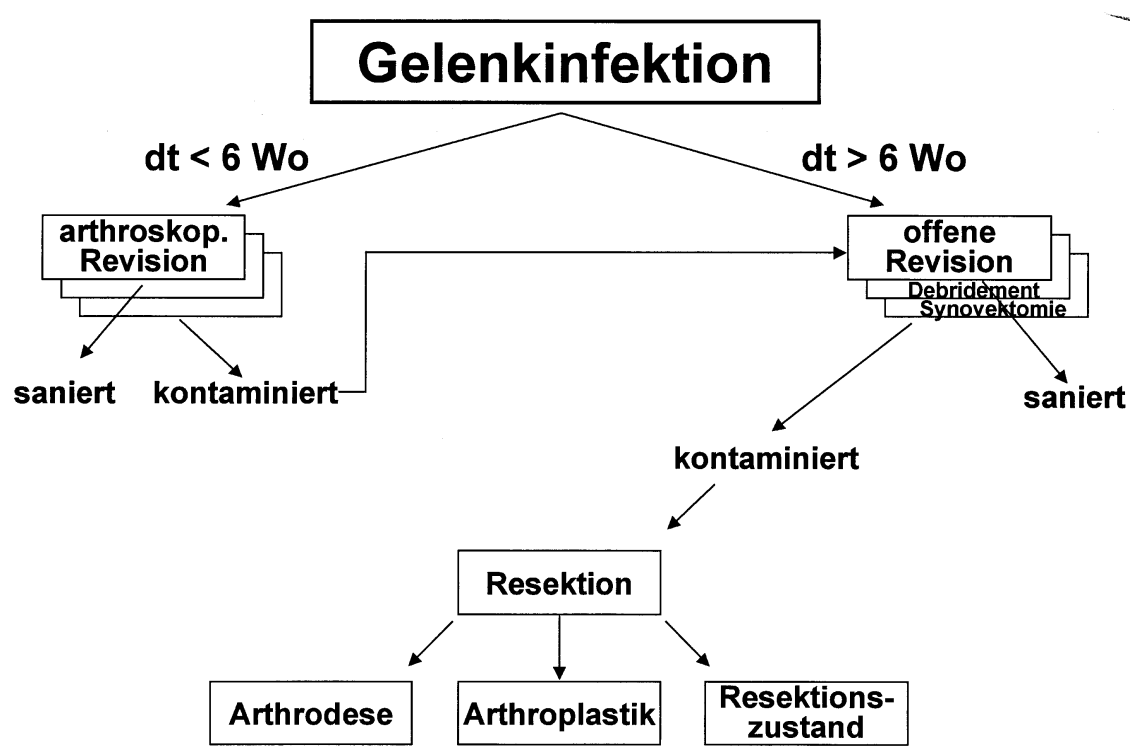

Abb. $1 \Delta$ Behandlungsalgorithmus bei akuten und chronischen Gelenkinfektionen 


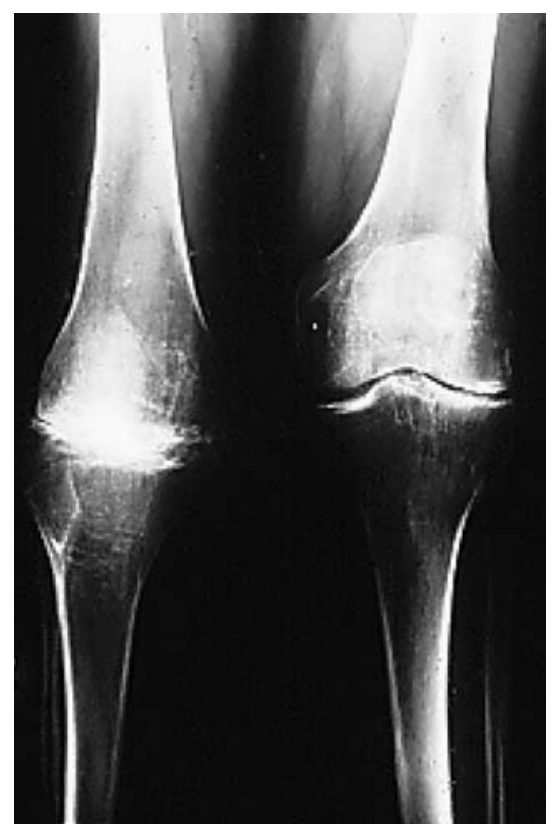

Abb. $2 \Delta$ Resektionszustand eines Kniegelenks nach infektbedingtem Knorpelverlust

Frühinfekts keinen Beitrag leisten, im Fall des Spätinfekts dokumentiert sie weit fortgeschrittene Veränderungen. Die Szintigraphie ist auch in der verfeinerten Form der granulozytenmarkierten Szintigraphie zu wenig spezifisch.

\section{Bakteriologische Untersuchung}

Einzig und allein die bakteriologische Untersuchung von Geweben aus dem betroffenen Gelenk hat letztendlich beweisenden Charakter einer Infektion. Allerdings muss auch hier mit einer nicht unerheblichen Quote an falsch-negativen Ergebnissen (10-16\%) gerechnet werden.

Die zusätzliche Histologie bestätigt beim Vorliegen von bakteriellen Entzündungen die granulomatöse Entzündungsreaktion. Große Hoffnungen für die Frühdiagnostik eines Infekts werden auf die molekularbiologischen Untersuchungen gesetzt. Die Fluoreszenz-basierte-insitu-Hybridisierung (FISH) ermöglicht den direkten Nachweis von Staphylococcus epidermidis und Staphylococcus aureus in Gewebeproben ohne vorherige Anzucht von Bakterien. Damit ließe sich in Zukunft im Fall einer vorliegenden Infektion eine nicht unwesentliche Zeitersparnis zwischen Infektverdacht und Behandlungsbeginn erreichen sowie der Nachweis einer Infektion auch in den Fällen führen, wo die konventionellen
Untersuchungsmethoden mittels Bakteriennachweis über Anzucht scheitern.

\section{Therapie}

Als Therapieziele bei den Gelenkinfektionen lässt sich eine klare Hierarchie der Prioritäten definieren:

1. Priorität: Infektsanierung

2. Priorität: Schmerzfreiheit

3. Priorität: Stabilität des Gelenks

4. Priorität: Mobilität des Gelenks

Bei der Therapie der Gelenkinfektionen folgen wir dem in Abb. 1 dargestellten Algorithmus.

\section{Frühinfekt}

Beim Frühinfekt, also binnen der ersten 6 Wochen, starten wir, wenn nicht andere Gründe dagegen sprechen, immer mit arthroskopischen Revisionen. In 3- bis 5-tägigem Abstand werden programmierte Revisionen mit ausgiebiger Lavage des Gelenks und systemischer testgerechter Antibiose durchgeführt. Wenn es nach 3 Arthroskopien gelingt, das Gelenk zu sanieren, werden keine weiteren Eingriffe durchgeführt. Ergibt die bakteriologische Untersuchung auch nach der 3. Arthroskopie noch eine kontaminierte Situation im Gelenkbinnenraum, wird zur offenen Revision übergegangen.

\section{Spätinfekt}

Eine Gelenkspätinfektion wird primär offen behandelt. Beim offenen Vorgehen erfolgen extra- und intraartikulär ein ausgedehntes Weichteildébridement, der Einsatz der Jet-Lavage, systemische und lokale, testgerechte Antibiose. Auch hier werden programmierte Revisionen in 3- bis 5-tägigem Abstand durchgeführt. Gelingt es nach 3 offenen Revisionen nicht, den Infekt zu sanieren, werden die Knorpelflächen der Gelenke komplett reseziert. Das betroffene Kniegelenk muss dann mittels einer Transfixierung am Fixateur externe ruhig gestellt werden. Nach Beherrschung der Infektion ergeben sich in der weiteren Behandlung 3 Optionen:

- Arthrodese

- Arthroplastik

- Resektionszustand

Während des gesamten therapeutischen Verlaufs kommt der Physiotherapie und der Ergotherapie eine entscheidende Schlüsselrolle für die Wiedererlangung der späteren Funktion des Gelenks zu. Auch unter diesem Gesichtspunkt der begleitenden und anschließenden Phy-
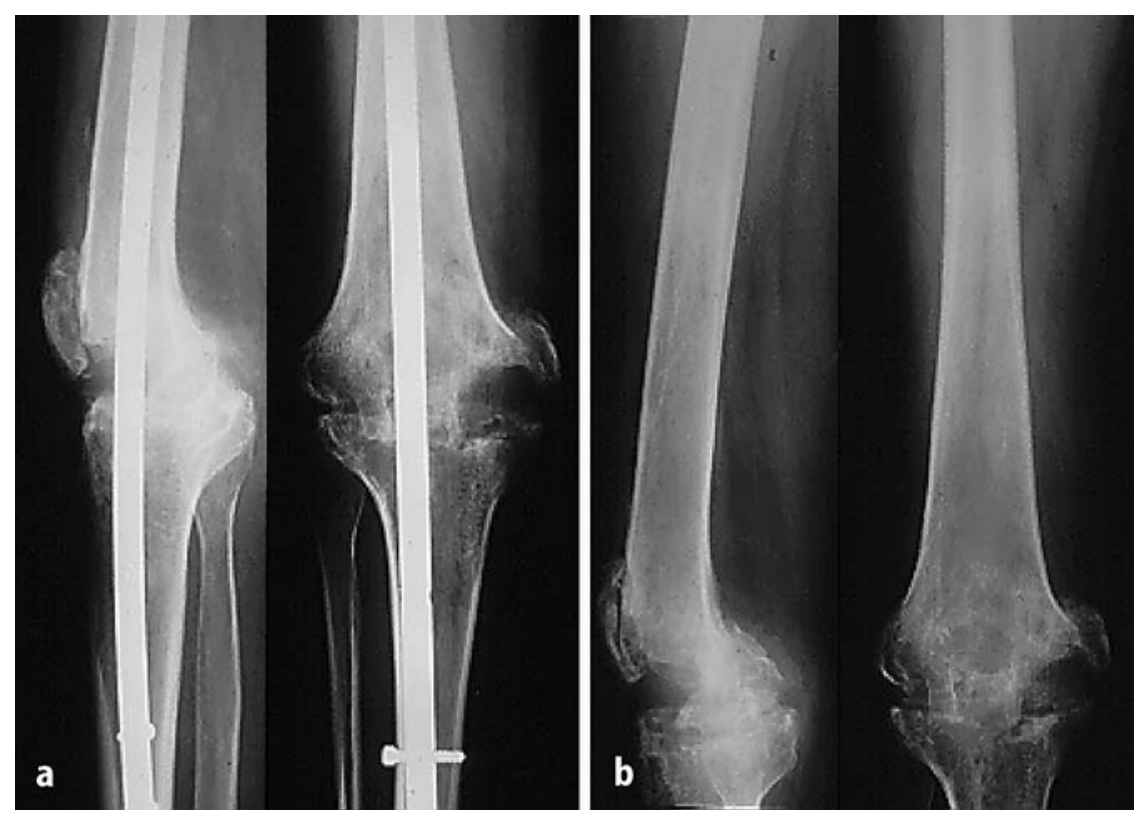

Abb. 3a,b $\Delta$ Arthrodese eines Kniegelenks nach Infektgonarthrose, a mit einliegendem Arthrodesenmarknagel, $b$ nach ME und erfolgter knöcherner Durchbauung 

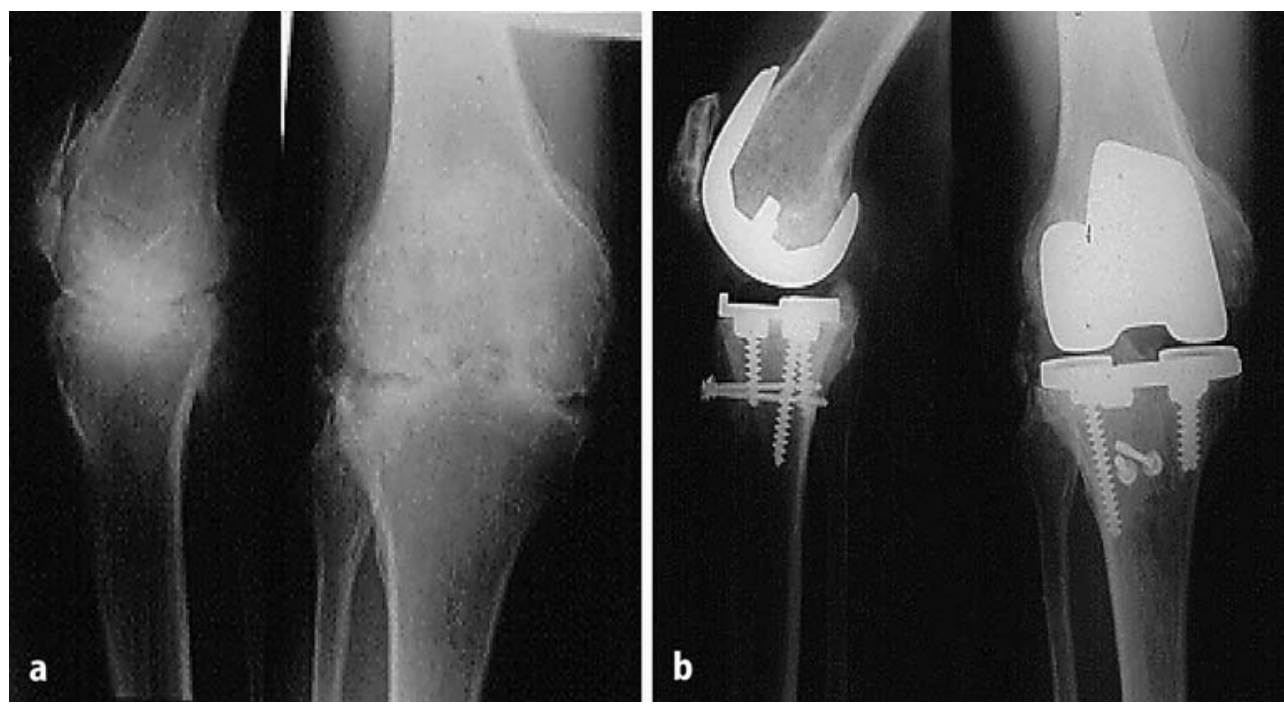

Abb. 4a,b $<$ Infektgonarthrose mit vollständigem Gelenkverschleiß bei einer 26-jährigen Patientin (a), Ersatz des zerstörten Gelenks durch eine bikondyläre, unzementierte Schlittenprothese (b)

siotherapie stellt die Behandlung von Gelenkinfektionen u. E. eine Zentrumsaufgabe dar.

Bei der Behandlung des Spätinfekts ist der irreversible Endpunkt mit dem Untergang des Knorpels erreicht. Diese Situation ist mit dem Fortbestehen eines dauerhaft funktionsfähigen Gelenks nicht vereinbar.

Im Gegensatz zur Girdlestone-Situation an der Hüfte kann das Belassen eines Resektionszustands am Kniegelenk eigentlich nur als historisch angesehen werden. Von Ausnahmen abgesehen bietet ein solcher Resektionszustand keine Alternative für den Patienten (Abb. 2).

Für alle Fälle, bei denen aus welchen Gründen auch immer, eine Endoprothese nicht in Frage kommen kann, stellt die Versteifung des Gelenks, die Arthrodese, die Therapie der Wahl dar (Abb. 3). $\mathrm{Ob}$ die Entscheidung für das technische Vorgehen einer Arthrodese dabei auf einen Fixateur externe inklusive Ringfixateur oder auf ein Nagelarthrodeseverfahren fällt, ist von zweitrangiger Bedeutung.

Die Kniegelenkendoprothetik stellt u. E. die oberste Behandlungsoption dar. Dabei sollte der Zeitraum zwischen Beherrschung der Infektion und Implantation der Endoprothese auf etwa 6-12 Wochen festgesetzt werden. Die ausreichende Exposition des Gelenks nach einer abgelaufenen Infektion mit Untergang des Knorpels ist dann meist nur durch eine Tuberositas-tibiae-Osteotomie möglich. Die Implantation sollte, wann immer möglich, in zementfreier Technik durchgeführt werden, wobei ein Patellarückflächenersatz anzustreben ist. Postoperativ wird der Patient für 3 Monate einer systemischen Antibiose unterzogen. Wichtig ist, den Patienten auf die eingeschränkte Ergebniserwartung aufmerksam zu machen.

Steht für den Patienten der Funktionserhalt des Gelenks absolut im Vordergrund, gibt es für die Indikationsstellung zur Endoprothese keine Altersgrenze, weder nach oben noch nach unten (Abb. 4).

\section{Schlussfolgerungen}

Zusammenfassend lässt sich schlussfolgern, dass auch bei persistierenden Infektionen am Kniegelenk die Frage eines Infektsanierungserfolgs ganz klar als Funktion des Faktors Zeit zu sehen ist. Dabei gelingt es in nahezu 100\% der Fälle, eine frühe Infektion zu beherrschen, in $2 / 3$ der Fälle bereits durch das arthroskopische Vorgehen, nur in $1 / 3$ der Fälle ist das nachgeschaltete offene Vorgehen notwendig.

Im Fall der Spätinfektion gelingt der Gelenkerhalt in $<20 \%$ der Fälle. Wird trotz des Verlusts der Knorpeloberfläche des Gelenks dennoch eine Infektsanierung erreicht, können 2/3 der Patienten erfolgreich durch eine Endoprothetik behandelt werden, nur in $1 / 3$ der Fälle ist die Arthrodese notwendig.

Zentral wichtig im gesamten Therapiekonzept der Gelenkinfektionen ist:

1. Den Infekt erkennen

2. Sich dazu bekennen

3. Schnell zu handeln

Durch Anwendung eines konsequenten Revisionsprotokolls analog dem oben angedeuteten Entscheidungsalgorithmus besteht im Fall des Frühinfekts die reale Chance auf einen Gelenkerhalt. Im Fall einer späten Gelenkinfektion werden gute Voraussetzungen für den endoprothetischen Ersatz des Gelenks geschaffen.

\section{Weiterführende Literatur}

1. Hofmann GO, Bär T, Bühren V (1997) Osteosyntheseimplantat und früher postoperativer Infekt: Sanierung mit oder ohne Materialentfernung? Chirurg 68: 1175-1180

2. Matava MJ, Evans TA, Wright RW, Shively RA (1998) Septic arthritis of the knee following anterior cruciate ligament reconstruction. Arthroscopy 14: 717-725

3. Schmidt J, Hackenbroch MH, Kumm D, Taravati $V(1996)$ Is instillation drainage for the treatment of infected joints, bones and soft tissues still up to date? Arch Orthop Trauma Surg 115: 149-152

4. Schneider U, Hierholzer G, Böhm HJ (1996) Knochen- und Gelenkinfektionen. Unfallchirurg 99: 789-800 